\title{
$\bullet$ Early Pulmonary Hypertension in COVID 19 Patient: A Case Report
}

\section{IJCRR}

Section: Healthcare

ISI Impact Factor

(2019-20): 1.628

IC Value (2019): 90.81

$\operatorname{SJIF}(2020)=7.893$

(c) (7) (8)

Copyright@IJCRR

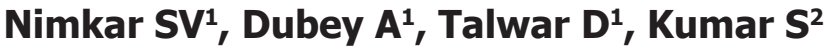

Junior Resident, Department of Medicine, Jawahar Lal Nehru Medical College, Datta Meghe Institute of Medical Sciences, Sawangi (Meghe), Wardha, India; ${ }^{P}$ Professor, Department of Medicine, Jawahar Lal Nehru Medical College, Datta Meghe Institute of Medical Sciences, Sawangi (Meghe), Wardha, India.

\section{ABSTRACT}

Introduction: This COVID 19 pandemic has altered the routine practices and the acute management of PAH patients. Patients with pre-existing $\mathrm{PAH}$ are very sensitive to the changes in their cardiopulmonary status.

Objective: To study a case of early pulmonary hypertension in COVID 19.

Case Report: A 45-year-old male presented with complaints of breathlessness at rest for 15 days which was gradually progressive. PCR was done and tested positive. The patient was treated for the same with negative PCR repeated report after 14 days and then again patient presented with the above complaints after 14 days of discharge. COVID-19 infection poses a bizarre threat to the vulnerable population with $\mathrm{PAH}$. The inpatient needs have been increased during the pandemic.

Observations: $\mathrm{PAH}$ patients had great difficulty in surviving the interventions which pose a significant cost during a pandemic that is stretching the health care system. So far, we have not seen many PAH patients with COVID-19 infections, and most of them have had favourable outcomes. This reflects the ability of these patients to isolate and quarantine themselves properly. Also, we are approaching having a successful treatment option or a vaccination for COVID-19 shortly.

Conclusion: In the current report, $\mathrm{PH}$ was associated with clinical, imaging and laboratory findings of more severe post-COVID-19 and with no morbid outcomes. A final decision is yet to be made. We believe it is important to screen for COVID-19 infection in all patients.

Key Words: COVID 19, Pulmonary hypertension, 2d echo, Mortality, Pandemic

\section{INTRODUCTION}

Pulmonary hypertension (PH) is primary (idiopathic) or secondary to a variety of underlying conditions like pulmonary, cardiovascular or systemic conditions. ${ }^{1,2}$ A prolonged elevation of pulmonary arterial pressure to more than $25 \mathrm{~mm} \mathrm{Hg}$ at rest or more than $30 \mathrm{~mm} \mathrm{Hg}$ with exercise, with mean pulmonary-capillary wedge pressure and left ventricular end-diastolic pressure of less than $15 \mathrm{~mm} \mathrm{Hg}$, is known as pulmonary arterial hypertension. ${ }^{3}$ Histologically, the appearance of lung tissue is similar in each condition: intimal fibrosis, increased medial thickness, pulmonary arterial occlusion, and plexiform lesions predominate. ${ }^{4}$

The COVID-19 pandemic has exhibited many peculiar challenges when caring for patients with pulmonary hypertension $(\mathrm{PH})$, particularly the patients with pre-existing pulmonary arterial hypertension (PAH), and chronic thromboembolic pulmonary hypertension (CTEPH). The presence of whether pre-existing $\mathrm{PH}$ or $\mathrm{PH}$ as a direct result of the lung injury that occurs post COVID-19 infection, or cardiomyopathy resulted from COVID-19 infection, or other comorbidity is likely to be a major contributor to the morbidity and mortality associated with COVID-19 infection.., 6

We report a case of a 45-year-old male with Bilateral Pleural Effusion with Mild ascites and Grade III Renal parenchymal disease (RPD) who had an asymptomatic course of COVID-19 infection who tested positive for infection by screening.

\section{CASE PRESENTATION}

A 45-year-old male presented with complaints of pedal oedema and distension of the abdomen for 6 days. He also

\section{Corresponding Author:}

Nimkar SV, Junior Resident, Department of Medicine, Jawahar Lal Nehru Medical College, Datta Meghe Institute of Medical Sciences, Sawangi (Meghe), Wardha, India; Phone: +917057632069; Email: shubhamnimkar7@gmail.com

ISSN: 2231-2196 (Print)

Received: 04.01 .2021
ISSN: 0975-5241 (Online)

Revised: 27.02 .2021
Accepted: 24.04 .2021
Published: 11.06 .2021 
had complaints of breathlessness at rest for 15 days which was gradually progressive. 14 days back On the previous admission, due to the current COVID-19 pandemic and as per our local strategy to eliminate COVID-19 infection among the population nasopharyngeal swab to screen for COVID-19, PCR was done and tested positive. Patient treated for same and PCR report repeated after 14 days it was negative (JNMC/COV/10175/2020) patient was discharged and then again patient presented with above complaints after 14 days discharge. On general examination: Pulse: 90/min, BP: 100/70mmHg, Oxygen saturation on room air was $95 \%$, respiratory rate: $24 / \mathrm{min}$. On abdominal examination: Distended abdomen with free fluid present in the abdomen, shifting dullness present. On respiratory examination: Dull percussion note, reduced breath sounds in both lung bases with bronchial breathing over the left infra-scapular area.

Blood investigations revealed-CBC investigations on cell count with PS showed 9.4 g/dL Hb, $31.2 \mathrm{~g} / \mathrm{dl} \mathrm{MCHC}, 72.5$ $\mathrm{p} \mathrm{MCV}$, and $22.6 \mathrm{pg} \mathrm{MCH}$. Total RBC count was 4.16 million $/ \mathrm{mm}^{3}, 7800 /$ microlitre, and 2.49/microlitre of blood. Prothrombin Time of 12.5 seconds, APTT 30.20 seconds, INR: 1.28.CK-MB value $29 \mathrm{IU} / \mathrm{L}$. random blood sugar $151 \mathrm{mg} / \mathrm{dL}$, Calcium: 6.g/dl, KFT showed Urea: $45 \mathrm{mg} /$ dl, Creatinine: $1.2 \mathrm{mg} / \mathrm{dl}$, Serum sodium: $146 \mathrm{mmol} / \mathrm{L}$, Serum potassium: $4.7 \mathrm{mmol} / \mathrm{L}$, LFT showed Serum albumin: $3.9 \mathrm{~g} / \mathrm{dl}$, alkaline phosphatase level was $55 \mathrm{IU} / \mathrm{L}$. Albumin $(3.9 \mathrm{~g} / \mathrm{dL})$, Bilirubin conjugated $(0.2 \mathrm{mg} / \mathrm{dL})$, bilirubin unconjugated $(0.7 \mathrm{mg} / \mathrm{dL})$, globulin $(3.8 \mathrm{~g} / \mathrm{dL})$, SGPT (50 U/L), SGOT (34 U/L), total protein $(7.7 \mathrm{~g} / \mathrm{dL})$, and total bilirubin $(0.9 \mathrm{mg} / \mathrm{dL})$ Lipid profile showed total cholesterol of $170 \mathrm{mg} / \mathrm{dL}$, triglycerides value was $102 \mathrm{mg} /$ $\mathrm{dL}$ dHDL, LDL, and VLDL values of $57 \mathrm{mg} / \mathrm{dL}, 93 \mathrm{mg} / \mathrm{dL}$, and $20 \mathrm{mg} / \mathrm{dL}$.Arterial blood gas (ABG) test showed 7.4 $\mathrm{pH}, 36.3 \mathrm{mmHg} \mathrm{PCO}$, and $110 \mathrm{mmHg} \mathrm{PO}$. Hepatitis $\mathrm{B} /$ Hepatitis C/ HIV status of this patient was negative (Figure 1).

2D Echo showed dilated right atrium, tricuspid regurgitant velocity $3.2 \mathrm{~m} / \mathrm{sec}$, systolic pulmonary artery pressure 44mmhg, left ventricle (LV), hypokinetic Inferior wall(IW) and Anterior wall (AW). Motion mode (M.mode) of 2D echo showed LVIDD value of $5.5 \mathrm{~cm}$, LVIDS value of $4.5 \mathrm{~cm}$, IVs value is 10 , posterior wall is 10 , EF value is $30 \%$, LA is $4.0 \mathrm{~cm}$. Dilated LV, severe pulmonary hypertension, mild AR, severe TR, Grade 3 diastolic dysfunction. IVC congested and non-collpsing (Figure 2).

A coronary angiography report showed mild coronary artery disease (CAD). HRCT scan of the thorax showed moderate pleural effusion on both sides with basal lung atelectasis with subsegmental collapse consolidation on the left basal lung. The severity score was $0 / 25$ indicating no obvious abnormality in bilateral lung fields.

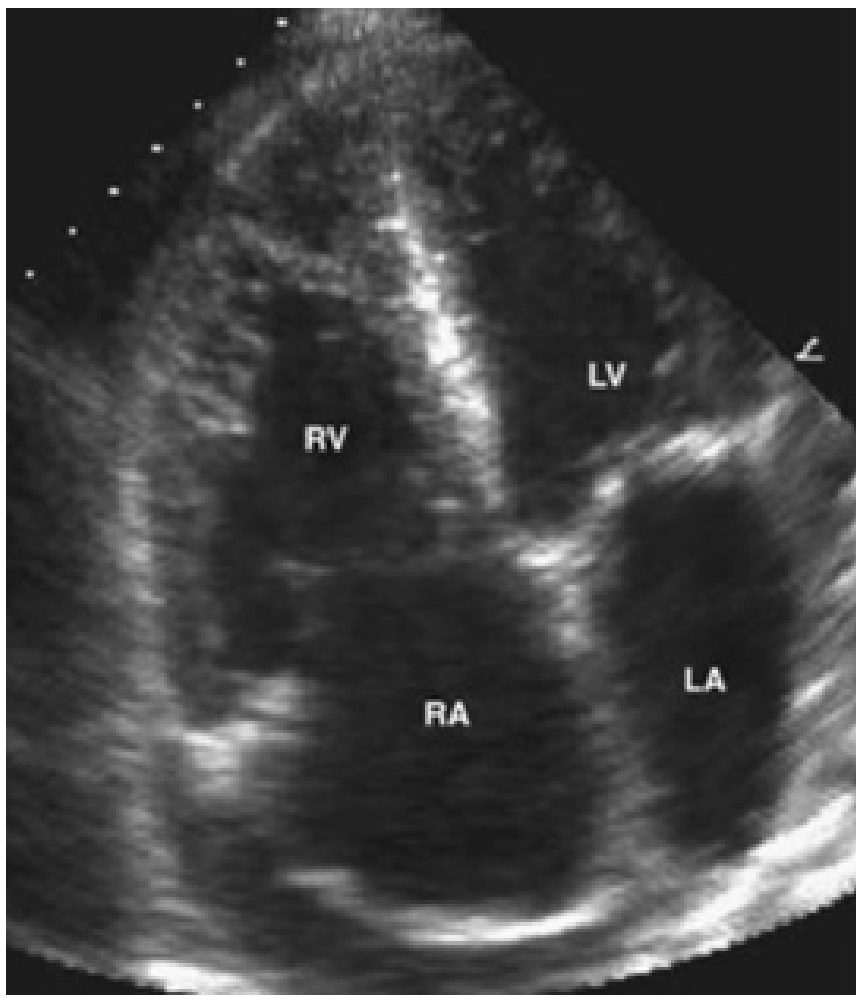

Figure 1: $2 \mathrm{~d}$ echo showed Dilated right atrium, tricuspid regurgitant velocity $3.2 \mathrm{~m} / \mathrm{sec}$.

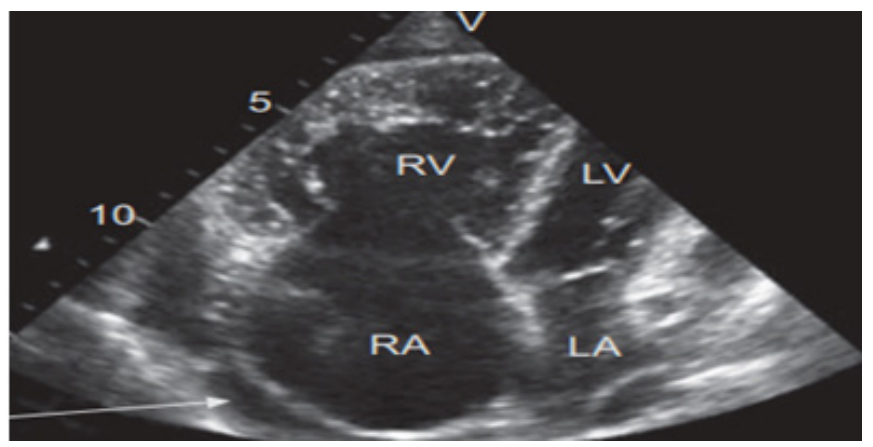

Figure 2: $2 \mathrm{~d}$ echo showed Dilated right atrium, tricuspid regurgitant velocity $3.2 \mathrm{~m} / \mathrm{sec}$.

The treatment regime followed for the patient during his hospital stay was as given:

- Injection Heparin 2500 IU

- Injection NTG $100 \mathrm{mcg}$

- Injection Dilzem 5mg

The patient was discharged after treatment and waiting for follow up.

\section{DISCUSSION}

Patients with pre-existing PAH are very sensitive to the changes in their cardiopulmonary status. Any interruption of care or the emergence of new cardiac or pulmonary pathology may result in death. In PAH, hospitalization is a risk 
factor for disease development. A newly available case series suggested that $86 \%$ of critically ill COVID-19-infected patients had heart failure and chronic kidney disease as the most common underlying medical conditions. ${ }^{7} \mathrm{PAH}$ patients have shown to have worse outcomes with all-cause hospitalizations. ${ }^{5}$ One should always keep in mind that COVID-19 infection may lead to hypoxemia leading to hypoxic pulmonary vasoconstriction, which increases pulmonary vascular resistance (PVR) resulting in decompensation of a high-risk PAH patient. ${ }^{7}$ Keep oxygen saturation above $92 \%$, continuing oral and parenteral therapies like phosphodiesterase-5 inhibitors (PDE5i), soluble guanylate cyclase stimulator (sGC), endothelin receptor antagonist (ERA), oral prostacyclin, oral prostaglandin I2 receptor agonist (PGI2) and intravenous or subcutaneous prostacyclins while treating with concurrent therapies for COVID-19 infection are recommended. As per institutional guidelines, continuing treatment with investigational therapies for COVID-19 infection along with the PAH treatment is also recommended.

COVID-19 infection poses a bizarre threat to the vulnerable population with PAH. PAH patients had great difficulty in surviving the interventions which posses a significant cost during a pandemic that is stretching the health care system. There is a shortage of ventilators during the COVID-19 pandemic, which poses even further concern for their utility in this population. A study done by Pagnesi et al. in 2020 stated that the prevalence of $\mathrm{PH}$ was $12.0 \%$. Patients with $\mathrm{PH}$ were older and had a higher burden of pre-existing cardiac comorbidities and signs of more severe acute respiratory syndrome in COVID-19 infection. ${ }^{8}$

Lee and colleagues in their study found that the cumulative incidence of recognized COVID-19 was similar to the general population. However, outcomes were worse, with a $50 \%$ rate of hospitalization and a $12 \%$ mortality rate in patients with PAH and CTEPH affected by COVID-19. The fact that incidence was higher in higher prevalence states seems to validate the conclusion that the rate is probably similar to the general population. ${ }^{9}$ A small survey and other COVID-19 $\mathrm{PAH} / \mathrm{CTEPH}$ series reported to date have suggested that COVID-19 has a relatively benign course in the setting of $\mathrm{PAH}$ and surprisingly favourable clinical outcomes. ${ }^{10-12}$

So far, we have not seen many PAH patients with COVID-19 infections, and most of them have had favourable outcomes. This reflects the ability of these patients to isolate and quarantine themselves properly. Also, we are approaching having a successful treatment option or a vaccination for COVID-19 shortly.

\section{CONCLUSION}

To our best knowledge, it is the first report of pulmonary hypertension in post-COVID 19 infections. We reported a smooth clinical course for COVID-19 infection in a male with Bilateral Pleural Effusion with Mild ascites and Grade III Renal parenchymal disease (RPD). In the current report, $\mathrm{PH}$ was associated with clinical, imaging and laboratory findings of more severe post-COVID-19 and with no morbid outcomes. One of the worthiest questions to ask nowadays is whether those patients should be considered high risk for COVID 19 infection and its complications during this pandemic or not. A final decision is yet to be made. We believe it is important to screen for COVID-19 infection in all patients.

\section{Acknowledgement: None}

\section{Conflict of Interest and Source of Funding: None}

\section{Author Contribution:}

Nimkar SV: Manuscript Preparation, Data Collection

Dubey A: Manuscript editing

Talwar D: Pliagrism Removal

Kumar S: Final editing and preparation according to journal guidelines

\section{REFERENCES}

1. Farber HW, Loscalzo J. Pulmonary arterial hypertension. N Engl J Med. 2004;351(16):1655-65.

2. Wiltshire E, Peña AS, MacKenzie K, Shaw G, Couper J. High dose folic acid is a potential treatment for pulmonary hypertension, including when associated with COVID-19 pneumonia. Med Hypoth. 2020;143:110142.

3. Simonneau G, Montani D, Celermajer DS, Denton CP, Gatzoulis MA, Krowka M, et al. Haemodynamic definitions and updated clinical classification of pulmonary hypertension. Eur Respiratory J. 2019;53(1):1801913.

4. Rubin LJ. Primary pulmonary hypertension. N Engl J Med 1997;336:111-7.

5. Ryan JJ, Melendres-Groves L, Zamanian RT, Oudiz RJ, Chakinala M, Rosenzweig EB, et al. Care of patients with pulmonary arterial hypertension during the coronavirus (COVID-19) pandemic. Pulm Circ. 2020;10(2):2045894020920153.

6. Jain A, DhruvTalwar SK. Spectrum of Respiratory Involvement in COVID 19 Era: An Overview. Indian J Forensic Med Toxicol. 2020;14(4):6593.

7. Sahay S, Farber HW. Management of hospitalized patients with pulmonary arterial hypertension and COVID-19 infection. Pulm Circ. 2020;10(3)1-5.

8. Pagnesi M, Baldetti L, Beneduce A, Calvo F, Gramegna M, Pazzanese V, et al. Pulmonary hypertension and right ventricular involvement in hospitalised patients with COVID-19. Heart. 2020;106(17):1324-31.

9. Lee JD, Burger CD, Delossantos GB, Grinnan D, Ralph DD, Rayner SG, et al. A survey-based estimate of COVID-19 incidence and outcomes among patients with pulmonary arterial hypertension or chronic thromboembolic pulmonary hypertension and impact on the process of care. Ann Am Thorac Soc 2020;17:1576-82.

10. Horn EM, Chakinala M, Oudiz R, Joseloff E, Rosenzweig EB. Could pulmonary arterial hypertension patients be at a lower risk from severe COVID-19? Pulm. Circ. 2020;10(2):1-2. 
11. Scuri P, Iacovoni A, Abete R, Cereda A, Grosu A, Senni M. An unexpected recovery of patients with pulmonary arterial hypertension and SARS-CoV-2 pneumonia: A case series. Pulm. Circ. 2020;10:204589402095658.
12. Nuche J, Pérez-Olivares C, De La Cal TS, López-Guarch CJ, Ynsaurriaga FA, Escribano-Subías P. Clinical course of COVID-19 in pulmonary arterial hypertension patients. Rev Esp Cardiol. 2020;73:775-778. 